Proyecciones Journal of Mathematics

Vol. 38, N $^{o}$ 1, pp. 59-82, March 2019.

Universidad Católica del Norte

Antofagasta - Chile

\title{
Algorithm for the generalized $\Phi$-strongly monotone mappings and application to the generalized convex optimization problem
}

\author{
M. O. Aibinu \\ University of Kwazulu-Natal, South Africa \\ and \\ O. T. Mewomo \\ University of Kwazulu-Natal, South Africa \\ Received : June 2017. Accepted : October 2018
}

\begin{abstract}
Let $E$ be a uniformly smooth and uniformly convex real Banach space and $E^{*}$ be its dual space. We consider a multivalued mapping $A$ : $E \rightarrow 2^{E^{*}}$ which is bounded, generalized $\Phi$-strongly monotone and such that for all $t>0$, the range $R\left(J_{p}+t A\right)=E^{*}$, where $J_{p}(p>1)$ is the generalized duality mapping from $E$ into $2^{E^{*}}$. Suppose $A^{-1}(0) \neq \emptyset$, we construct an algorithm which converges strongly to the solution of $0 \in$ Ax. The result is then applied to the generalized convex optimization problem.
\end{abstract}

Keywords : Generalized $\Phi$-strongly monotone, surjective property, generalized convex, optimization.

2000 Mathematics Subject Classification : $\quad 47 \mathrm{HO6}, 47 \mathrm{HO}$, 47J05, $47 \mathrm{~J} 25$. 


\section{Introduction}

Let $E$ be a real normed space, $E^{*}$ denotes its dual space and let $J_{p}(p>1)$ denote the generalized duality mapping from $E$ into $2^{E^{*}}$ given by

$$
J_{p}(x)=\left\{f \in E^{*}:\langle x, f\rangle=\|x\|^{p},\|f\|=\|x\|^{p-1}\right\},
$$

where $\langle.,$.$\rangle is the generalized duality pairing. For p=2$, the mapping $J_{2}$ is denoted by $J$ and it is called the normalized duality mapping from $E$ into $2^{E^{*}}$. If $E$ is smooth, then $J_{p}$ is single-valued and onto if $E$ is reflexive. For a uniformly smooth Banach space $E$ with $J_{p}: E \rightarrow E^{*}$ and $J_{q}^{*}: E^{*} \rightarrow E$ being the duality mappings with gauge functions $\nu(t)=t^{p-1}$ and $\nu(s)=s^{q-1}$ respectively, $J_{p}^{-1}=J_{q}^{*}$ (see e.g., Alber and Ryazantseva, p. 36 [42], Cioranescu [10], p. 25-77, Xu and Roach [40], Zălinescu [42]).

The problem of finding zero points for maximal monotone operators plays an important role in optimizations because it can be reduced to a convex minimization problem and a variational inequality problem. The approximation of solutions of these problems has also been studied by numerous authors (see for examples, [1, 22, 23, 30, 31, 41].

Let $E$ be a real normed space and $A: E \rightarrow 2^{E^{*}}$ a multivalued mapping. $A$ is called monotone if for each $x, y \in E$, the following inequality holds:

$$
\left\langle x^{*}-y^{*}, x-y\right\rangle \geq 0 \quad \forall x^{*} \in A x, \quad y^{*} \in A y .
$$

$A$ is said to satisfy the range condition if in addition, $R\left(J_{p}+t A\right)$ (the range of $\left.\left(J_{p}+t A\right)\right)$ is all of $E^{*}$ for all $t>0$. $A$ is called maximal monotone if it is monotone and its graph is not properly contained in the graph of any other monotone mapping. $A$ is said to be generalized $\Phi$-strongly monotone if there exists a strictly increasing function $\Phi:[0, \infty) \rightarrow[0, \infty)$ with $\Phi(0)=0$ such that

$$
\left\langle x^{*}-y^{*}, x-y\right\rangle \geq \Phi(\|x-y\|) \quad \forall x^{*} \in A x, \quad y^{*} \in A y .
$$

The mapping $A$ is $\phi$-strongly monotone if there exists a strictly increasing function $\phi:[0, \infty) \rightarrow[0, \infty)$ with $\phi(0)=0$ such that

$$
\left\langle x^{*}-y^{*}, x-y\right\rangle \geq\|x-y\| \phi(\|x-y\|) \quad \forall x^{*} \in A x, \quad y^{*} \in A y,
$$

and it is strongly monotone if there exists a constant $k \in(0,1)$ such that

$$
\left\langle x^{*}-y^{*}, x-y\right\rangle \geq k\|x-y\|^{2} \forall x^{*} \in A x, \quad y^{*} \in A y .
$$


Clearly, the class of strongly monotone mappings is a subclass of $\phi$-strongly monotone mappings (by taking $\phi(t)=k t$ ) and the class of $\phi$-strongly monotone mappings is a subclass of generalized $\Phi$-strongly monotone mappings (by taking $\Phi(t)=t \phi(t)$ ). It is well known that the class of generalized $\Phi$-strongly monotone mappings is the largest, among the classes of monotone-type mappings such that if a solution of the equation $0 \in A x$ exists, it is necessarily unique. We recall some important generalized monotonicity properties which have been studied for multivalued mappings. Let $E$ be a real topological vector space and $E^{*}$ be the dual space. Suppose $K \subseteq E$ is a nonempty subset of $E$ and $A: K \rightarrow 2^{E^{*}}$ is a multivalued mapping. For each $x, y \in K, A$ is said to be respectively pseudomonotone and quasimonotone (see e.g., Karamardian and Schaible [25], Karamardian et al. [26]), if for any $x^{*} \in A(x), y^{*} \in A(y)$, the following implications hold:

$$
\left\langle y^{*}, x-y\right\rangle \geq 0 \Rightarrow\left\langle x^{*}, x-y\right\rangle \geq 0,
$$

and

$$
\left\langle y^{*}, x-y\right\rangle>0 \Rightarrow\left\langle x^{*}, x-y\right\rangle \geq 0 .
$$

Also, $A$ is said to be quasimonotone if

$$
\min \left\{\left\langle x^{*}, x-y\right\rangle,\left\langle y^{*}, x-y\right\rangle\right\} \leq 0 .
$$

The two definitions of quasimonotonicity coincide (see e.g., [33]). It is clear that a monotone mapping is pseudomonotone, while a pseudomonotone mapping is quasimonotone. The converse is not necessarily true. In the case of a single-valued linear mapping $A$ defined on $E$ (where $E:=R^{n}$ ), for $\alpha \in E^{*} \backslash\{0\}$, it is known that if $A+\alpha$ is quasimonotone, then $A$ is monotone (see e.g., [26]). This result has been extended by several authors (see, e.g., Hadjisavvas [17], He [20], Isac and Motreanu [21]). The theory of monotone multivalued mappings is nowadays well developed. Results on generic single-valuedness and upper semicontinuity have been settled several decades ago.

The concept of quasimonotone multivalued mapping is younger. It broadly generalizes monotone mappings (see e.g., Aussel and Fabian [8], Phelps [34]). Quasimonotone mappings are closely related to the so-called demand functions in mathematical economics (see e.g., Levin [29], Karlin [27] for more details). Let $E$ and $F$ be two real topological vector spaces, $\theta_{F}$ denotes the zero vector of $F, K$ is a nonempty convex subset of $E$ and $T: K \rightarrow L(E, F)$ is a set-valued mapping, where $L(E, F)$ denotes the space of all continuous linear mappings from $E$ into $F$. For $x$ and $y$ in $K$, we 
recall from [15] that $S \subseteq L(E, F)$ is said to have the surjective property on $[x, y]=\{x+t(y-x): t \in[0,1]\}$ ( for short, on $x$ and $y$ ), whenever the following equality holds:

$$
\langle S, x-y\rangle:=\left\{\left\langle x^{*}, x-y\right\rangle=x^{*}(x-y): x^{*} \in S\right\}=F,
$$

where $\left\langle x^{*}, x-y\right\rangle=x^{*}(x-y)$ denotes the value of $x^{*}$ at $(x-y)$. Let $K \subseteq E$ be a nonempty set and $S \subseteq L(E, F), S$ is said to have the surjective property on $K$, if for every $x \in K$, there exists $y \in K$ such that $S$ has the surjective property on $x$ and $y$. For $x, y \in K$, consider $x-y$ as a linear functional (denoted by $\widehat{x-y}$ ) on $L(E, F)$ as follows:

$$
\langle\widehat{x-y}, f\rangle=\langle f, x-y\rangle
$$

where $f \in L(E, F)$. Thus, the surjective property of $S \subseteq L(E, F)$ on $x, y$ implies that the image of $S$ under the linear functional $\widehat{x-y}$ is $F$. For more details on this, (see Farajzadeh and Plubtieng [15]).

Classical examples of quasimonotone mappings are the subdifferentials of lower semicontinuous quasiconvex functions. The interest in quasimonotone mapping stems mainly from the fact that the derivative and more generally, the subdifferential of a quasiconvex function is quasimonotone. This is similar to the link between convex functions and monotonicity of their (generalized) derivative (see, Aussel et al. [6], [7] for more details). A subset $K$ of $E$ is said to be convex if for every $x, y \in K$, and $\lambda \in[0,1]$, we have

$$
\lambda x+(1-\lambda) y \in K .
$$

A function $f: K \rightarrow R$ defined on a convex subset $K$ of $E$ is convex if for any $x, y \in K$ and $\lambda \in[0,1]$, we have

$$
f(\lambda x+(1-\lambda) y) \leq \lambda f(x)+(1-\lambda) f(y) .
$$

If we have strict inequality for all $x \neq y$ in the above definition, the function is said to be strictly convex. The first type of generalized convex function was considered by De Finetti [13]. However, he did not name this class of functions. The term "quasiconvex function" was given subsequently by Fenchel [16] after six years. A function $f: K \rightarrow R$ is quasiconvex if

$$
f(\lambda x+(1-\lambda) y) \leq \max \{f(x), f(y)\}, \quad \forall x, y \in K \text { and } \lambda \in[0,1] .
$$

Clearly every convex function is quasiconvex but the converse is not always true. Just consider the function $f: R \rightarrow R$ defined by

$$
f(x)=\left\{\begin{array}{cc}
x-1, & x \leq 1 \\
\ln x, & x>1
\end{array}\right.
$$


Then $f$ is quasiconvex but it is not convex. In fact it is concave. A function $f: E \rightarrow R \cup\{+\infty\}$ is convex if and only if for each $\alpha \in E^{*}$ the function $u \mapsto$ $f(u)+\langle\alpha, u\rangle$ is quasiconvex. A classical tool to study lower semicontinuous functions is the Clarke subdifferential. Let $f: E \rightarrow R \cup\{+\infty\}$ be a lower semicontinuous function. The Clarke subdifferential of $f$ is the operator $\partial f: E \rightarrow 2^{E^{*}}$ defined for each $u \in E$ by

$$
\partial f(u)= \begin{cases}\left\{u^{*} \in E^{*}:\left\langle u^{*}, v\right\rangle \leq f^{\uparrow}(u ; v), \quad \forall v \in E\right\}, & \text { if } u \in \operatorname{domf} \\ \emptyset, & \text { if } u \notin \operatorname{domf},\end{cases}
$$

where

$$
f^{\uparrow}(u ; v):=\sup _{\epsilon>0} \inf _{\substack{\gamma>0 \\ \delta>0 \\ \lambda>0}} \sup _{\substack{x \in B_{\gamma}(u) \\ f(x) \leq f(u)+\delta \\ t \in(0, \lambda)}} \inf _{y \in B_{\epsilon}(v)} \frac{f(x+t y)-f(x)}{t}
$$

is the Rockafellar directional derivative (see e.g., Aussel et al. [7], Clarke [11], pp. 308, Rockafellar [37]). It is known as an axiom of a subdifferential that if $f$ attains at $u$ a local minimum, then $0 \in \partial f(u)$ (see e.g., J. P. Penot [32]). A function $\psi:[0, \infty) \rightarrow[0, \infty)$ such that $\psi$ is nondecreasing, $\psi(0)=0$ and $\psi$ is continuous at 0 is called a modulus of continuity. It follows that a mapping $A: X \rightarrow Y$ is uniformly continuous if and only if it has a modulus of continuity, where $X$ and $Y$ are real normed linear spaces. Recall that a function having a bounded set range is called a bounded function and given a convex function $f$, if $u \in$ int $\operatorname{dom} f$ then $\partial f(u)$ is nonempty and bounded.

For 2-uniformly convex real Banach space with uniformly Gâteaux differentiable norm, Diop et al. [12] studied the class of strongly monotone mappings and applied their result to the convex minimization problem. Chidume and Idu [9] considered the class of maximal monotone mappings in uniformly convex and uniformly smooth Banach spaces and obtained the minimizer of a convex function defined from a Banach space $E$ to $R$.

Motivated and inspired by the above results and the ongoing research in this direction, in this paper, we consider the largest class of monotone mappings in uniformly smooth and uniformly convex Banach spaces. We study the class of the generalized $\Phi$-strongly monotone mappings which is the largest classes of monotone-type mappings such that if a solution of the equation $0 \in A x$ exists, it is necessarily unique. Assuming existence, we construct an algorithm for the solution of the equation $0 \in A x$ and apply our result to the generalized convex optimization problem. 


\section{Preliminaries}

Let $E$ be a real normed space of dimension greater or equal to 2 and let $S:=\{x \in E:\|x\|=1\}$. E is said to have a Gâteaux differentiable norm (or $E$ is called smooth), if the limit

$$
\lim _{t \rightarrow 0} \frac{\|x+t y\|-\|x\|}{t}
$$

exists for each $x, y \in S$, E is Fréchet differentiable, if it is smooth and the limit is attained uniformly for $y \in S$. Further, $E$ is said to be uniformly smooth, if it is smooth and the limit is attained uniformly for each $x, y \in S$. The modulus of smoothness of $E$, denoted by $\rho_{E}$ is defined by

$$
\rho_{E}(\tau)=\sup \left\{\frac{\|x+y\|+\|x-y\|}{2}-1,\|x\|=1, \quad\|y\|=\tau\right\}, \tau \geq 0 .
$$

Clearly, $\rho_{E}(\tau) \leq \tau$ for all $\tau \geq 0$. $E$ is said to be uniformly smooth if $\lim _{\tau \rightarrow 0^{+}} \frac{\rho_{E}(\tau)}{\tau}=0$. The modulus of convexity of $E, \delta_{E}:(0,2] \rightarrow[0,1]$ is defined by

$$
\delta_{E}(\epsilon)=\inf \left\{1-\frac{\|x+y\|}{2}:\|x\|=\|y\|=1,\|x-y\|>\epsilon\right\} .
$$

$E$ is uniformly convex if and only if $\delta_{E}(\epsilon)>0$ for every $\epsilon \in(0,2]$. A normed linear space $E$ is said to be strictly convex if

$$
\|x\|=\|y\|=1, x \neq y \Rightarrow \frac{\|x+y\|}{2}<1 .
$$

Every uniformly convex space is strictly convex. Typical examples of uniformly smooth and uniformly convex spaces are $L_{p}, l_{p}, W_{p}^{m}$ spaces for $1<p<\infty$ and Hilbert spaces.

Definition 2.1. Let $E$ be a smooth real Banach space with dual $E^{*}$.

(i) The function $\phi: E \times E \rightarrow R$ is defined by

$$
\phi(x, y)=\|x\|^{2}-2\langle x, J y\rangle+\|y\|^{2}, \text { for all } x, y \in E,
$$

where $J$ is the normalized duality map from $E$ to $E^{*}$, introduced by Alber and has been studied by Alber [4], Kamimura and Takahashi [24], and Reich [35]. 
(ii) The function $\phi_{p}: E \times E \rightarrow R$ is defined by

$$
\phi_{p}(x, y)=\frac{p}{q}\|x\|^{q}-p\left\langle x, J_{p} y\right\rangle+\|y\|^{p}, \text { for all } x, y \in E,
$$

where $J_{p}$ is the generalized duality map from $E$ to $E^{*}, p$ and $q$ are real numbers such that $q \geq p>1$ and $\frac{1}{p}+\frac{1}{q}=1$.

(iii) The mapping $V_{p}: E \times E^{*} \rightarrow R$ is defined by

$$
\begin{gathered}
V_{p}\left(x, x^{*}\right)=\frac{p}{q}\|x\|^{q}-p\left\langle x, x^{*}\right\rangle+\left\|x^{*}\right\|^{p} \quad \forall x \in E, x^{*} \in E^{*} \text { such that } \\
q \geq p>1, \frac{1}{p}+\frac{1}{q}=1 .
\end{gathered}
$$

Remark 2.2. These remarks follow from Definition 2.1:

(i) For $p=2, \phi_{2}(x, y)=\phi(x, y)$. Also, it is obvious from the definition of the function $\phi_{p}$ that

$$
(\|x\|-\|y\|)^{p} \leq \phi_{p}(x, y) \leq(\|x\|+\|y\|)^{p} \text { for all } x, y \in E \text {. }
$$

(ii) It is obvious that

$$
V_{p}\left(x, x^{*}\right)=\phi_{p}\left(x, J_{p}^{-1} x^{*}\right) \quad \forall x \in E, x^{*} \in E^{*} .
$$

We need the following lemmas and theorems in the sequel.

Lemma 2.3. [3]. Let $E$ be a smooth uniformly convex real Banach space. For $d>0$, let $B_{d}(0):=\{x \in E:\|x\| \leq d\}$. Then for arbitrary $x, y \in B_{d}(0)$,

$$
\|x-y\|^{p} \geq \phi_{p}(x, y)-\frac{p}{q}\|x\|^{q}, \quad q \geq p>1, \quad \frac{1}{p}+\frac{1}{q}=1 .
$$

Lemma 2.4. [3]. Let $E$ be a smooth uniformly convex real Banach space with $E^{*}$ as its dual. Then,

$$
V_{p}\left(x, x^{*}\right)+p\left\langle J_{p}^{-1} x^{*}-x, y^{*}\right\rangle \leq V_{p}\left(x, x^{*}+y^{*}\right)
$$

for all $x \in E$ and $x^{*}, y^{*} \in E^{*}$. 
Lemma 2.5. [3]. Let $E$ be a reflexive strictly convex and smooth real Banach space with the dual $E^{*}$. Then,

$\phi_{p}(y, x)-\phi_{p}(y, z) \geq p\left\langle z-y, J_{p} x-J_{p} z\right\rangle=p\left\langle y-z, J_{p} z-J_{p} x\right\rangle \quad$ for all $x, y, z \in E$.

Lemma 2.6. [14]. Let $E$ be a real topological vector space, $K$ a nonempty convex subset of $E$ and $A: K \rightarrow 2^{E^{*}}$ a multivalued mapping. Assume $S \subseteq E^{*}$ is connected and has the surjective property on $K$. If $A+\alpha$ is quasimonotone for all $\alpha \in S$, then $A$ is monotone on $K$.

Lemma 2.7. [42]. Let $\psi: R^{+} \rightarrow R^{+}$be increasing with $\lim _{t \rightarrow \infty} \psi(t)=\infty$. Then $J_{\psi}^{-1}$ is single-valued and uniformly continuous on bounded sets if and only if $E$ is a uniformly convex Banach space.

Theorem 2.8. [38]. Let $E$ be a real uniformly convex Banach space. For arbitrary $r>0$, let $B_{r}(0):=\{x \in E:\|x\| \leq r\}$. Then, there exists a continuous strictly increasing convex function

$$
g:[0, \infty) \rightarrow[0, \infty), \quad g(0)=0,
$$

such that for every $x, y \in B_{r}(0), j_{p}(x) \in J_{p}(x), j_{p}(y) \in J_{p}(y)$, the following inequalities hold:

(i) $\|x+y\|^{p} \geq\|x\|^{p}+p\left\langle y, j_{p}(x)\right\rangle+g(\|y\|) ;$

(ii) $\left\langle x-y, j_{p}(x)-j_{p}(y)\right\rangle \geq g(\|x-y\|)$.

Lemma 2.9. [39]. Let $\left\{a_{n}\right\}$ be a sequence of nonnegative real numbers satisfying the following relations:

$$
a_{n+1} \leq\left(1-\alpha_{n}\right) a_{n}+\alpha_{n} \sigma_{n}+\gamma_{n}, \quad n \in N,
$$

where

(i) $\{\alpha\}_{n} \subset(0,1), \sum_{n=1}^{\infty} \alpha_{n}=\infty$;

(ii) $\lim \sup \{\sigma\}_{n} \leq 0$;

(iii) $\gamma_{n} \geq 0, \sum_{n=1}^{\infty} \gamma_{n}=\infty$. 
Then, $a_{n} \rightarrow 0$ as $n \rightarrow \infty$.

Lemma 2.10. [36]. Let $E$ be a reflexive smooth Banach space and let $A$ be a monotone operator from $E$ to $E^{*}$. Then $A$ is maximal if and only if $R(J+t A)=E^{*}$ for all $t>0$. That is, every maximal monotone map satisfies the range condition.

Theorem 2.11. [28]. Let $E^{*}$ be a real strictly convex dual Banach space with a Fréchet differentiable norm and let $A: E \rightarrow E^{*}$ be monotone and $R(J+t A)=E^{*}$ for some $t>0$. Suppose $A^{-1} 0 \neq \emptyset$, then for every $x \in E$, the $\lim _{t \rightarrow \infty} J_{t} x:=\lim _{t \rightarrow \infty}(J+t A)^{-1} x$ converges strongly to $P x$, where $P$ is the nearest point retraction of $E$ onto $A^{-1} 0$.

Lemma 2.12. [24]. Let $E$ be a smooth uniformly convex real Banach space and let $\left\{x_{n}\right\}$ and $\left\{y_{n}\right\}$ be two sequences from $E$. If either $\left\{x_{n}\right\}$ or $\left\{y_{n}\right\}$ is bounded and $\phi\left(x_{n}, y_{n}\right) \rightarrow 0$ as $n \rightarrow \infty$, then $\left\|x_{n}-y_{n}\right\| \rightarrow 0$ as $n \rightarrow \infty$.

Lemma 2.13. [9]. Let $E$ be an arbitrary real normed space and $E^{*}$ be its dual space. Let $A: E \rightarrow 2^{E^{*}}$ be any mapping. Then $A$ is monotone if and only if $T:=(J-A): E \rightarrow 2^{E^{*}}$ is $J$-pseudocontractive.

Lemma 2.14. [7]. Let $f: E \rightarrow R \cup\{+\infty\}$ be a lower semicontinuous functional on a Banach space $E$. Then, $\partial f$ is quasimonotone if and only if $f$ is quasiconvex.

\section{Main Results}

Theorem 3.1. Let $E$ be a uniformly smooth and uniformly convex real Banach space and $E^{*}$ be its dual space. Let $A: E \rightarrow 2^{E^{*}}$ be a multivalued mapping which is bounded, generalized $\Phi$-strongly monotone and such that for all $t>0$, the range $R\left(J_{p}+t A\right)=E^{*}$ and $A^{-1}(0) \neq \emptyset$. Let $\left\{\lambda_{n}\right\}$ and $\left\{\theta_{n}\right\}$ be real sequences in $(0,1)$ such that,

(i) $\lim \theta_{n}=0$ and $\left\{\theta_{n}\right\}$ is decreasing;

(ii) $\sum_{n=1}^{\infty} \lambda_{n} \theta_{n}=\infty$;

(iii) $\lim _{n \rightarrow \infty}\left(\left(\theta_{n-1} / \theta_{n}\right)-1\right) / \lambda_{n} \theta_{n}=0, \sum_{n=1}^{\infty} \lambda_{n}^{2}<\infty$. 
For arbitrary $x_{1} \in E$, define $\left\{x_{n}\right\}$ iteratively by:

$x_{n+1}=J_{p}^{-1}\left(J_{p} x_{n}-\lambda_{n}\left(\mu_{n}^{x}+\theta_{n}\left(J_{p} x_{n}-J_{p} x_{1}\right)\right)\right), \quad \mu_{n}^{x} \in A x_{n} \quad n \in N$,

where $J_{p}$ is the generalized duality mapping from $E$ into $E^{*}$. There exists a real constant $\gamma_{0}>0$ such that $\psi_{*}\left(\lambda_{n} M_{0}\right) \leq \gamma_{0}, \quad n \in N$ for some constant $M_{0}>0$. Then the sequence $\left\{x_{n}\right\}$ converges strongly to the solution of $0 \in A x$.

Proof. We divide the proof into two parts.

Part 1: We prove that $\left\{x_{n}\right\}$ is bounded. Let $x^{*} \in E$ be a solution of $0 \in A x$. It suffices to show that there exists $r>0$ such that $\phi_{p}\left(x^{*}, x_{n}\right) \leq$ $r, \forall n \in N$. Let $\psi_{*}$ be the modulus of continuity of $J_{p}^{-1}: E^{*} \rightarrow E$ on bounded sets of $E^{*}$ and $\delta \in(0,1)$ be arbitrary but fixed. Let $r>0$ be such that

$$
r \geq \max \left\{\phi_{p}\left(x^{*}, x_{1}\right), \delta^{p}+\frac{p}{q}\left\|x^{*}\right\|^{q}\right\} .
$$

The proof is by induction. By construction, $\phi_{p}\left(x^{*}, x_{1}\right) \leq r$. Suppose that $\phi_{p}\left(x^{*}, x_{n}\right) \leq r$ for some $n \in N$. We show that $\phi_{p}\left(x^{*}, x_{n+1}\right) \leq r$. Suppose this is not the case, then $\phi_{p}\left(x^{*}, x_{n+1}\right)>r$.

From inequality $(2.2)$, we have $\left\|x_{n}\right\| \leq r^{\frac{1}{p}}+\left\|x^{*}\right\|$. Let $B:=\left\{x \in E: \phi_{p}\left(x^{*}, x\right) \leq r\right\}$ and since $A$ is bounded, we define $M_{0}:=\sup \left\{\left\|\mu^{x}+\theta_{n}\left(J_{p} x-J_{p} x_{1}\right)\right\|: \theta_{n} \in(0,1), x \in B, \mu^{x} \in A x\right\}+1$.

Let $\psi_{*}:[0, \infty) \rightarrow[0, \infty)$ be the modulus of continuity of $J_{p}^{-1}$. Observe that by the uniform continuity of $J_{p}^{-1}$ on bounded subsets of $E^{*}$, for all $\mu_{n}^{x} \in A x_{n}$, we have

$$
\left\|J_{p}^{-1}\left(J_{p} x_{n}\right)-J_{p}^{-1}\left(J_{p} x_{n}-\lambda_{n}\left(\mu_{n}^{x}+\theta_{n}\left(J_{p} x_{n}-J_{p} x_{1}\right)\right)\right)\right\| \leq \psi_{*}\left(\lambda_{n} M_{0}\right) .
$$

Define

$$
\gamma_{0}:=\min \left\{1, \frac{\Phi\left(\frac{\delta}{2}\right)}{2 M_{0}}\right\} \text { where } \psi_{*}\left(\lambda_{n} M_{0}\right) \leq \gamma_{0} \text { and } \psi_{*}\left(\lambda_{n} M_{0}\right) \geq \frac{\delta}{2}
$$


Applying Lemma 2.4 with $y^{*}:=\lambda_{n}\left(\mu_{n}^{x}+\theta_{n}\left(J_{p} x_{n}-J_{p} x_{1}\right)\right)$ and by using the definition of $x_{n+1}$, we compute as follows,

$$
\begin{array}{r}
\phi_{p}\left(x^{*}, x_{n+1}\right)=\phi_{p}\left(x^{*}, J_{p}^{-1}\left(J_{p} x_{n}-\lambda_{n}\left(\mu_{n}^{x}+\theta_{n}\left(J_{p} x_{n}-J_{p} x_{1}\right)\right)\right)\right) \\
=V_{p}\left(x^{*}, J_{p} x_{n}-\lambda_{n}\left(\mu_{n}^{x}+\theta_{n}\left(J_{p} x_{n}-J_{p} x_{1}\right)\right)\right) \\
\leq V_{p}\left(x^{*}, J_{p} x_{n}\right) \\
-p \lambda_{n}\left\langle J_{p}^{-1}\left(J_{p} x_{n}-\lambda_{n}\left(\mu_{n}^{x}+\theta_{n}\left(J_{p} x_{n}-J_{p} x_{1}\right)\right)\right)-x^{*}, \mu_{n}^{x}+\theta_{n}\left(J_{p} x_{n}-J_{p} x_{1}\right)\right\rangle \\
=\phi_{p}\left(x^{*}, x_{n}\right)-p \lambda_{n}\left\langle x_{n}-x^{*}, \mu_{n}^{x}+\theta_{n}\left(J_{p} x_{n}-J_{p} x_{1}\right)\right\rangle \\
-p \lambda_{n}\left\langle J_{p}^{-1}\left(J_{p} x_{n}-\lambda_{n}\left(\mu_{n}^{x}+\theta_{n}\left(J_{p} x_{n}-J_{p} x_{1}\right)\right)\right)-x_{n}, \mu_{n}^{x}+\theta_{n}\left(J_{p} x_{n}-J_{p} x_{1}\right)\right\rangle .
\end{array}
$$

By Schwartz inequality and uniform continuity property of $J_{p}^{-1}$ on bounded sets of $E^{*}$ (Lemma 2.7), we obtain

$$
\begin{aligned}
\phi_{p}\left(x^{*}, x_{n+1}\right) \leq & \phi_{p}\left(x^{*}, x_{n}\right)-p \lambda_{n}\left\langle x_{n}-x^{*}, \mu_{n}^{x}+\theta_{n}\left(J_{p} x_{n}-J_{p} x_{1}\right)\right\rangle \\
& +p \lambda_{n} \psi_{*}\left(\lambda_{n} M_{0}\right) M_{0}(\text { By applying inequality }(3.4)) \\
\leq & \phi_{p}\left(x^{*}, x_{n}\right)-p \lambda_{n}\left\langle x_{n}-x^{*}, \mu_{n}^{x}-\mu_{*}^{x}\right\rangle \quad\left(\text { for } \mu_{*}^{x} \in A x^{*}\right. \\
& \text { since } \left.x^{*} \in N(A)\right) \\
& -p \lambda_{n} \theta_{n}\left\langle x_{n}-x^{*}, J_{p} x_{n}-J_{p} x_{1}\right\rangle+p \lambda_{n} \psi_{*}\left(\lambda_{n} M_{0}\right) M_{0} .
\end{aligned}
$$

By Lemma 2.5, $p\left\langle x^{*}-x_{n}, J_{p} x_{n}-J_{p} x_{1}\right\rangle \leq \phi_{p}\left(x^{*}, x_{n}\right)-\phi_{p}\left(x^{*}, x_{1}\right) \leq 0$. Also, since $A$ is generalized $\Phi$-strongly monotone, we have,

$$
\begin{array}{r}
\phi_{p}\left(x^{*}, x_{n+1}\right) \\
\leq \phi_{p}\left(x^{*}, x_{n}\right)-p \lambda_{n} \Phi\left(\left\|x_{n}-x^{*}\right\|\right)-p \lambda_{n} \theta_{n}\left\langle x_{n}-x^{*}, J_{p} x_{n}-J_{p} x_{1}\right\rangle+p \lambda_{n} \psi_{*}\left(\lambda_{n} M_{0}\right) M_{0} \\
=\phi_{p}\left(x^{*}, x_{n}\right)-p \lambda_{n} \Phi\left(\left\|x_{n}-x^{*}\right\|\right)+p \lambda_{n} \theta_{n}\left\langle x^{*}-x_{n}, J_{p} x_{n}-J_{p} x_{1}\right\rangle+p \lambda_{n} \psi_{*}\left(\lambda_{n} M_{0}\right) M_{0} \\
(3.5) \quad \leq \phi_{p}\left(x^{*}, x_{n}\right)-p \lambda_{n} \Phi\left(\left\|x_{n}-x^{*}\right\|\right)+p \lambda_{n} \psi_{*}\left(\lambda_{n} M_{0}\right) M_{0} .
\end{array}
$$
have

By the uniform continuity property of $J_{p}^{-1}$ on bounded sets of $E^{*}$, we

$$
\left\|x_{n+1}-x_{n}\right\|=\left\|J^{-1}\left(J x_{n+1}\right)-J^{-1}\left(J x_{n}\right)\right\| \leq \psi_{*}\left(\lambda_{n} M_{0}\right),
$$

such that

$$
\left\|x_{n+1}-x^{*}\right\|-\left\|x_{n}-x^{*}\right\| \leq \psi_{*}\left(\lambda_{n} M_{0}\right)
$$

which gives

$$
\left\|x_{n}-x^{*}\right\| \geq\left\|x_{n+1}-x^{*}\right\|-\psi_{*}\left(\lambda_{n} M_{0}\right) .
$$


From Lemma 2.3,

$$
\begin{aligned}
\left\|x_{n+1}-x^{*}\right\|^{p} & \geq \phi_{p}\left(x^{*}, x_{n+1}\right)-\frac{p}{q}\left\|x^{*}\right\| \\
& \geq r-\frac{p}{q}\left\|x^{*}\right\| \\
& \geq\left(\delta^{p}+\frac{p}{q}\left\|x^{*}\right\|\right)-\frac{p}{q}\left\|x^{*}\right\| \\
& \geq \delta^{p} .
\end{aligned}
$$

So,

$$
\left\|x_{n+1}-x^{*}\right\| \geq \delta
$$

Therefore, the inequality (3.6) becomes,

$$
\begin{aligned}
\left\|x_{n}-x^{*}\right\| & \geq \delta-\psi_{*}\left(\lambda_{n} M_{0}\right) \\
& \geq \frac{\delta}{2} .
\end{aligned}
$$

Thus,

$$
\Phi\left(\left\|x_{n}-x^{*}\right\|\right) \geq \Phi\left(\frac{\delta}{2}\right) .
$$

Substituting (3.7) into (??) gives

$$
\begin{aligned}
r<\phi_{p}\left(x^{*}, x_{n+1}\right) & \leq \phi_{p}\left(x^{*}, x_{n}\right)-p \lambda_{n} \Phi\left(\frac{\delta}{2}\right)+p \lambda_{n} \psi_{*}\left(\lambda_{n} M_{0}\right) M_{0} \\
& \leq \phi_{p}\left(x^{*}, x_{n}\right)-p \lambda_{n} \Phi\left(\frac{\delta}{2}\right)+p \lambda_{n} \gamma_{0} M_{0} \\
& \leq \phi_{p}\left(x^{*}, x_{n}\right)-\frac{p \lambda_{n}}{2} \Phi\left(\frac{\delta}{2}\right) \\
& =r-\frac{p \lambda_{n}}{2} \Phi\left(\frac{\delta}{2}\right)<r
\end{aligned}
$$

a contradiction. Hence, $\phi_{p}\left(x^{*}, x_{n+1}\right) \leq r$. By induction, $\phi_{p}\left(x^{*}, x_{n}\right) \leq$ $r \forall n \in N$. Thus, from inequality (2.2), $\left\{x_{n}\right\}$ is bounded.

Part 2: We now show that $\left\{x_{n}\right\}$ converges strongly to a solution of $0 \in A x$. Since $A$ is generalized $\Phi$-strongly monotone and the range $R\left(J_{p}+\right.$ $t A)=E^{*}$, by the strict convexity of $E$, we obtain for every $t>0$, and $x \in E$, there exists a unique $x_{t} \in D(A)$, where $D(A)$ is the domain of $A$ such that

$$
J_{p} x \in J_{p} x_{t}+t A x_{t}
$$


If $J_{p_{t}} x=x_{t}$, then we can define a single-valued mapping $J_{p_{t}}: E \rightarrow D(A)$ by $J_{p_{t}}=\left(J_{p}+t A\right)^{-1} J_{p}$. Such a $J_{p_{t}}$ is called the resolvent of $A$. Therefore, by Theorem 2.11, for each $n \in N$, there exists a unique $y_{n} \in D(A)$ such that

$$
y_{n}=\left(J_{p}+\frac{1}{\theta_{n}} A\right)^{-1} J_{p} x_{1} .
$$

Then, we have $\left(J_{p}+\frac{1}{\theta_{n}} A\right) y_{n}=J_{p} x_{1}$, such that

$$
\theta_{n}\left(J_{p} y_{n}-J_{p} x_{1}\right)+\mu_{n}^{y}=0, \quad \mu_{n}^{y} \in A y_{n} .
$$

Observe that the sequence $\left\{y_{n}\right\}$ is bounded because it is a convergent sequence by Theorem 2.11. Moreover, $\left\{x_{n}\right\}$ is bounded and hence $\left\{A x_{n}\right\}$ is bounded. Following the same arguments as in part 1, we get,

$\phi_{p}\left(y_{n}, x_{n+1}\right) \leq \phi_{p}\left(y_{n}, x_{n}\right)-p \lambda_{n}\left\langle x_{n}-y_{n}, \mu_{n}^{x}+\theta_{n}\left(J_{p} x_{n}-J_{p} x_{1}\right)\right\rangle+p \lambda_{n} \gamma_{0} M_{0}$.

By the generalized $\Phi$-strongly monotonicity of $A$ and using Theorem 2.8 and Eq. (3.8), we obtain,

$$
\begin{array}{r}
\left\langle x_{n}-y_{n}, \mu_{n}^{x}+\theta_{n}\left(J_{p} x_{n}-J_{p} x_{1}\right)\right\rangle \\
=\left\langle x_{n}-y_{n}, \mu_{n}^{x}+\theta_{n}\left(J_{p} x_{n}-J_{p} y_{n}+J_{p} y_{n}-J_{p} x_{1}\right)\right\rangle \\
=\theta_{n}\left\langle x_{n}-y_{n}, J_{p} x_{n}-J_{p} y_{n}\right\rangle+\left\langle x_{n}-y_{n}, \mu_{n}^{x}+\theta_{n}\left(J_{p} y_{n}-J_{p} x_{1}\right)\right\rangle \\
=\theta_{n}\left\langle x_{n}-y_{n}, J_{p} x_{n}-J_{p} y_{n}\right\rangle+\left\langle x_{n}-y_{n}, \mu_{n}^{x}-\mu_{n}^{y}\right\rangle \\
\geq \theta_{n} g\left(\left\|x_{n}-y_{n}\right\|\right)+\Phi\left(\left\|x_{n}-y_{n}\right\|\right)
\end{array}
$$

$\geq \frac{1}{p} \theta_{n} \phi_{p}\left(y_{n}, x_{n}\right)$ (by Lemma 2.3 for some real constants $p>1$ ).

Therefore, the inequality (3.9) becomes

$$
\phi_{p}\left(y_{n}, x_{n+1}\right) \leq\left(1-\lambda_{n} \theta_{n}\right) \phi_{p}\left(y_{n}, x_{n}\right)+p \lambda_{n} \gamma_{0} M_{0} .
$$

Observe that by Lemma 2.5, we have

$$
\begin{aligned}
\phi_{p}\left(y_{n}, x_{n}\right) & \leq \phi_{p}\left(y_{n-1}, x_{n}\right)-p\left\langle y_{n}-x_{n}, J_{p} y_{n-1}-J_{p} y_{n}\right\rangle \\
& =\phi_{p}\left(y_{n-1}, x_{n}\right)+p\left\langle x_{n}-y_{n}, J_{p} y_{n-1}-J_{p} y_{n}\right\rangle \\
& \leq \phi_{p}\left(y_{n-1}, x_{n}\right)+\left\|J_{p} y_{n-1}-J_{p} y_{n}\right\|\left\|x_{n}-y_{n}\right\| .
\end{aligned}
$$


Let $R>0$ such that $\left\|x_{1}\right\| \leq R,\left\|y_{n}\right\| \leq R$ for all $n \in N$. We obtain from Eq.(3.8) that

$$
J_{p} y_{n-1}-J_{p} y_{n}+\frac{1}{\theta_{n}}\left(\mu_{n-1}^{y}-\mu_{n}^{y}\right)=\frac{\theta_{n-1}-\theta_{n}}{\theta_{n}}\left(J_{p} x_{1}-J_{p} y_{n-1}\right) .
$$

By taking the duality pairing of each side of this equation with respect to $y_{n-1}-y_{n}$ and by the generalized $\Phi$-strongly monotonicity of $A$, we have

$$
\left\langle J_{p} y_{n-1}-J_{p} y_{n}, y_{n-1}-y_{n}\right\rangle \leq \frac{\theta_{n-1}-\theta_{n}}{\theta_{n}}\left\|J_{p} x_{1}-J_{p} y_{n-1}\right\|\left\|y_{n-1}-y_{n}\right\|,
$$

which gives,

$$
\left\|J_{p} y_{n-1}-J_{p} y_{n}\right\| \leq\left(\frac{\theta_{n-1}}{\theta_{n}}-1\right)\left\|J_{p} y_{n-1}-J_{p} x_{1}\right\| .
$$

Using (3.11) and (3.12), the inequality (3.10) becomes

$$
\phi_{p}\left(y_{n}, x_{n+1}\right) \leq\left(1-\lambda_{n} \theta_{n}\right) \phi_{p}\left(y_{n-1}, x_{n}\right)+C\left(\frac{\theta_{n-1}}{\theta_{n}}-1\right)+p \lambda_{n} \gamma_{0} M_{0},
$$

for some constant $C>0$. By Lemma $2.9, \phi_{p}\left(y_{n-1}, x_{n}\right) \rightarrow 0$ as $n \rightarrow 0$ and using Lemma 2.12, we have that $x_{n}-y_{n-1} \rightarrow 0$ as $n \rightarrow 0$. Since by Theorem 2.11, $y_{n} \rightarrow x^{*} \in N(A)$, we obtain that $x_{n} \rightarrow x^{*}$.

Corollary 3.2. [3]. Let $E$ be a uniformly smooth and uniformly convex real Banach space and $E^{*}$ be its dual space. Suppose $A: E \rightarrow E^{*}$ is bounded, $\eta$-strongly monotone and satisfies the range condition such that $A^{-1}(0) \neq \emptyset$. Let $\left\{\lambda_{n}\right\}$ and $\left\{\theta_{n}\right\}$ be real sequences in $(0,1)$ such that,

(i) $\lim \theta_{n}=0$ and $\left\{\theta_{n}\right\}$ is decreasing;

(ii) $\sum_{n=1}^{\infty} \lambda_{n} \theta_{n}=\infty$;

(iii) $\lim _{n \rightarrow \infty}\left(\left(\theta_{n-1} / \theta_{n}\right)-1\right) / \lambda_{n} \theta_{n}=0, \quad \sum_{n=1}^{\infty} \lambda_{n}^{2}<\infty$.

For arbitrary $x_{1} \in E$, define $\left\{x_{n}\right\}$ iteratively by:

$$
x_{n+1}=J_{p}^{-1}\left(J_{p} x_{n}-\lambda_{n}\left(A x_{n}+\theta_{n}\left(J_{p} x_{n}-J_{p} x_{1}\right)\right)\right), n \in N
$$

where $J_{p}$ is the generalized duality mapping from $E$ into $E^{*}$. There exists a real constant $\epsilon_{0}>0$ such that $\psi\left(\lambda_{n} M_{0}\right) \leq \epsilon_{0}, \quad n \in N$ for some constant $M_{0}>0$. Then, the sequence $\left\{x_{n}\right\}$ converges strongly to the solution of $A x=0$. 
Proof. Take $\Phi(\|x-y\|):=\eta\|x-y\|^{p}$ in Theorem 3.1, then the desired result follows.

Corollary 3.3. [9]. Let $E$ be a uniformly convex and uniformly smooth real Banach space and $E^{*}$ be its dual space. Let $T: E \rightarrow 2^{E^{*}}$ be a $J$ pseudocontractive and bounded map such that $(J-T)$ is maximal monotone. Suppose $F_{E}^{J}(T)=\{v \in E: J v \in T v\} \neq \emptyset$. For arbitrary $x_{1}, u \in E$, define a sequence $\left\{x_{n}\right\}$ iteratively by:

$$
x_{n+1}=J^{-1}\left(\left(1-\lambda_{n}\right) J x_{n}+\lambda_{n} \eta_{n}-\lambda_{n} \theta_{n}\left(J x_{n}-J u\right)\right), \eta_{n} \in T x_{n}, \quad n \in N,
$$

where $\left\{\lambda_{n}\right\}$ and $\left\{\theta_{n}\right\}$ are real sequences in $(0,1)$ satisfying the following conditions:

(i) $\sum_{n=1}^{\infty} \lambda_{n} \theta_{n}=\infty$,

(i) $\lambda_{n} M_{0}^{*} \leq \gamma_{0} \theta_{n} ; \delta_{E}^{-1}\left(\lambda_{n} M_{0}^{*}\right) \leq \gamma_{0} \theta_{n}$,

(iii) $\frac{\delta_{E}^{-1}\left(\frac{\theta_{n-1}-\theta_{n}}{\theta_{n}} K\right)}{\lambda_{n} \theta_{n}} \rightarrow 0 ; \frac{\delta_{E^{*}}^{-1}\left(\frac{\theta_{n-1}-\theta_{n}}{\theta_{n}} K\right)}{\lambda_{n} \theta_{n}} \rightarrow 0$ as $n \rightarrow \infty$,

(iv) $\frac{1}{2} \frac{\theta_{n-1}-\theta_{n}}{\theta_{n}} K \in(0,1)$,

for some constants $M_{0}^{*}>0$ and $\gamma_{0}>0$, where $\delta_{E}:(0, \infty) \rightarrow(0, \infty)$ is the modulus of convexity of $E$ and $K:=4 R L \sup \{\|J x-J y\|:\|x\| \leq R,\|y\| \leq R\}+$ $1, \quad x, y \in E, \quad R>0$. Then the sequence $\left\{x_{n}\right\}$ converges strongly to a $J$ fixed point of $T$.

Proof. Define $A:=(J-T)$, then by the Lemma 2.13, $A$ is a bounded maximal monotone map. Therefore, the iterative sequence (3.14) is equivalent to

$$
x_{n+1}=J^{-1}\left(J x_{n}-\lambda_{n}\left(A x_{n}+\theta_{n}\left(J x_{n}-J u\right)\right)\right), n \in N,
$$

where $J$ is the normalized duality mapping from $E$ into $E^{*}$. Since the generalized $\Phi$-strongly monotone implies monotone, the result follows from Lemma 2.10 and by Theorem 3.1 . 
Corollary 3.4. [2]. Let $E$ be a $p$-uniformly convex real Banach space with uniformly Gâteaux differentiable norm such that $\frac{1}{p}+\frac{1}{q}=1, p \geq 2$ and $E^{*}$ its dual space. Let $A: E \rightarrow E^{*}$ be a bounded and $\eta$-strongly monotone mapping such that $A^{-1} 0 \neq \emptyset$. For arbitrary $x_{1} \in E$, let $\left\{x_{n}\right\}$ be the sequence defined iteratively by

$$
x_{n+1}=J_{p}^{-1}\left(J_{p} x_{n}-\lambda_{n} A x_{n}\right), n \in N,
$$

where $J_{p}$ is the generalized duality mapping from $E$ into $E^{*}$ and $\left\{\lambda_{n}\right\} \subset$ $\left(0, \gamma_{0}\right), \gamma_{0} \leq 1$ is a real sequence satisfying the following conditions:

(i) $\sum_{n=1}^{\infty} \lambda_{n}=\infty$;

(ii) $\sum_{n=1}^{\infty} \lambda_{n}^{2}<\infty$

Then, the sequence $\left\{x_{n}\right\}$ converges strongly to the unique point $x^{*} \in A^{-1} 0$.

Proof. Observe that uniformly convex and uniformly smooth real Banach spaces are more general than the $p$-uniformly convex real Banach spaces with uniformly Gâteaux differentiable norm. Also, by taking $\theta_{n}=0$ in Theorem 3.1, we obtain the desired result.

Corollary 3.5. [12]. Let $E$ be a 2-uniformly convex real Banach space with uniformly Gâteaux differentiable norm and $E^{*}$ its dual space. Let $A: E \rightarrow E^{*}$ be a bounded and $k$-strongly monotone mapping such that $A^{-1} 0 \neq \emptyset$. For arbitrary $x_{1} \in E$, let $\left\{x_{n}\right\}$ be the sequence defined iteratively by:

$$
x_{n+1}=J^{-1}\left(J x_{n}-\alpha_{n} A x_{n}\right), n \in N,
$$

where $J$ is the normalized duality mapping from $E$ into $E^{*}$ and $\left\{a_{n}\right\} \subset$ $(0,1)$ is a real sequence satisfying the following conditions:

(i) $\sum_{n=1}^{\infty} \alpha_{n}=\infty$;

(ii) $\sum_{n=1}^{\infty} \alpha_{n}^{2}<\infty$.

Then, there exists $\gamma_{0}>0$ such that if $\alpha_{n}<\gamma_{0}$, the sequence $\left\{x_{n}\right\}$ converges strongly to the unique solution of the equation $A x=0$. 
Proof. By taking $p=2$ in Corollary 3.4, we obtain the desired result.

\section{Application to the generalized convex optimization prob- lem}

Let $E$ be a real Banach space with the dual $E^{*}$ and $A$, a multivalued mapping from $E$ into $2^{E^{*}}$. According to Hassouni [18], for $K$ subset of $E$, and $\bar{x} \in K, A$ satisfies the variational inequality below if and only if

$$
\forall x \in K,\left\langle\mu^{x}, x-\bar{x}\right\rangle \geq 0, \quad \forall \mu^{x} \in A x .
$$

Consider now the quasiconvex minimization problem

$$
\min _{x \in K} f(x)
$$

where $f: E \rightarrow R \cup\{+\infty\}$ is lower semicontinuous and quasiconvex. Let $N$ be a convex open neighborhood of $\bar{x}$. The necessary and sufficient condition to obtain a solution of 4.2 is given in the Lemma 4.1 below.

Lemma 4.1. [19]. If $K=N$ or $K=E$, then following assertions are equivalent:

(i) $\bar{x}$ is an optimal solution of (4.2),

(ii) $\partial f$ satisfies (4.1).

Remark 4.2. For any single-valued quasimonotone operator $\partial f$, the operator $h(x):=\{\alpha \partial f(x): \alpha \geq 0\}$ is also quasimonotone and $\operatorname{Gr}(\partial f) \subset G r(h)$ provided $\partial f \neq 0$, where $G r(\partial f)$ and $G r(h)$ denote the graph of $\partial f$ and of $h$ respectively. It follows that for every non-constant smooth quasiconvex function $f$, the single-valued quasimonotone operator $\partial f$ is not maximal (see e.g., Levin [29]).

Next, we give a useful definition and establish a lemma which is necessary in establishing our main result in this section. Let $E$ be a real topological vector space, $K$ a nonempty convex subset of $E, A: K \rightarrow L(E, R)=2^{E^{*}}$ a multivalued mapping and $S \subseteq 2^{E^{*}}$.

Definition 4.3. A multivalued mapping $A: K \rightarrow 2^{E^{*}}$ is said to have the surjective property if the range of $A$ excluding the zero vector (i.e 
$R(A) \backslash\{0\})$ has the surjective property. Indeed, suppose $S$ has the surjective property on $K$ and $f \in R(A) \backslash\{0\}$, then a multivalued mapping foS $\subset L(E, R)=2^{E^{*}}$ is said to have the surjective property on $K$ provided

$$
\langle f o S, x-y\rangle:=\left\{\left\langle f o x^{*}, x-y\right\rangle: x^{*} \in S\right\}=R .
$$

Lemma 4.4. Let $E$ be a uniformly smooth and uniformly convex real Banach space, $K$ a nonempty convex subset of $E$ and $A: K \rightarrow 2^{E^{*}}$ a multivalued mapping. Suppose $S \subseteq 2^{E^{*}}$ is connected and has the surjective property on $K$. Then $A$ satisfies the range condition if and only if for each $\alpha \in S, A+\alpha$ is quasimonotone and has the surjective property on $K$.

Proof. $\quad \Rightarrow "$ Suppose $A$ satisfies the range condition. That is, $A$ is monotone and $R\left(J_{p}+t A\right)=E^{*}$ for all $t>0$. Therefore for each $\alpha \in X^{*}$, the operator $u \mapsto A(u)+\alpha$ is obviously monotone, hence quasimonotone. Next, suppose for contradiction that $A+\alpha$ has no surjective property, that is $\exists x \in K$, a convex subset of $E$ such that $\forall y \in K$

$$
\langle A+\alpha, x-y\rangle=\left\{\left\langle\text { fou }^{*}+\alpha, x-y\right\rangle, u^{*}, \alpha \in S\right\} \neq R .
$$

It follows that for each $u^{*} \in S$, the range of

$$
g(t):=-t\left\langle f o u^{*}, x-y\right\rangle-\left\langle u^{*}, x-y\right\rangle
$$

is not equal to $R$. Recall that monotonicity of $A$ gives that

$$
\left\langle x^{*}-y^{*}, x-y\right\rangle \geq 0 \Rightarrow\left\langle x^{*}, x-y\right\rangle \geq\left\langle y^{*}, x-y\right\rangle \quad \forall x^{*} \in A x, \quad y^{*} \in A y .
$$

Therefore, there exists $t_{0} \in R$ such that

$$
\left\langle x^{*}, x-y\right\rangle \geq-t_{0}\left\langle\text { fou }^{*}, x-y\right\rangle-\left\langle u^{*}, x-y\right\rangle \geq\left\langle y^{*}, x-y\right\rangle .
$$

Setting $\alpha:=t_{0}$ fou $^{*}+u^{*}$, we deduce that

$$
\left\langle x^{*}+\alpha, x-y\right\rangle \geq 0,
$$

while

$$
\left\langle y^{*}+\alpha, x-y\right\rangle \leq 0 .
$$

Thus contradicting the pseudomonotonicity and hence quasimonotonicity of the map $A+\alpha$.

$" \Leftarrow$ "Suppose that $A+\alpha$ is quasimonotone and has the surjective property. We show that $A$ satisfies the range condition. By Lemma 2.6, $A$ is monotone 
since $A+\alpha$ is quasimonotone. Next is to show that $R\left(J_{p}+t A\right)=E^{*}$ for all $t>0$. Since $A+\alpha$ has the surjective property on $K$, for every $u^{*} \in R\left(J_{p}+t A\right)$, the line $L=\left\{u^{*}+t f o u^{*}: t \in R^{+}\right\}$has surjective property on $K$. But $L \subset E^{*}$, therefore

$$
R\left(J_{p}+t A\right) \subseteq E^{*} .
$$

Also, for a given $u^{*} \in S$ and each $v^{*} \in E^{*}$, define

$$
\left\langle v^{*}, x-y\right\rangle=\left\langle u^{*}+t f o u^{*}, y-x\right\rangle
$$

for every $x, y \in K$. Therefore, $v^{*}:=u^{*}+t$ fou $u^{*} \in R\left(J_{p}+t A\right)$. Hence $R\left(J_{p}+t A\right)=E^{*}$

Theorem 4.5. Let $E$ be a uniformly smooth and uniformly convex real Banach space with dual space $E^{*}$ and $S \subseteq 2^{E^{*}}$ is connected and has the surjective property. Let $f: E \rightarrow R \cup\{+\infty\}$ be a bounded lower semicontinuous quasiconvex function with nonempty interior. Suppose for each $\alpha \in S$, $\partial f+\alpha$ is quasimontone and has the surjective property with $(\partial f)^{-1} 0 \neq \emptyset$. Then, for arbitrary $x_{1} \in E$, the iteration $\left\{x_{n}\right\}$ defined by

$$
x_{n+1}=J_{p}^{-1}\left(J_{p} x_{n}-\lambda_{n}\left((\partial f) x_{n}+\theta_{n}\left(J_{p} x_{n}-J_{p} x_{1}\right)\right)\right), n \in N .
$$

converges strongly to some $x^{*} \in(\partial f)^{-1} 0$.

Proof. $\quad f$ is a bounded quasiconvex function with nonempty interior, therefore by Lemma 2.14, $\partial f$ is a bounded quasimonotone operator. Also, $0 \in \partial f(u)$ if and only if $f$ attains at $u$ a local minimum. By Lemma 4.4, $\partial f$ satisfies the range condition. Thus, the result follows from Theorem 3.1.

Remark 4.6. Prototype for our iteration parameters in Theorem 3.1 are, $\lambda_{n}=\frac{1}{(n+1)^{a}}$ and $\theta_{n}=\frac{1}{(n+1)^{b}}$, where $0<b<a$ and $a+b<1$.

Conclusion 4.7. Most of the existing results on the approximation of solutions of monotone-type mappings have been proved in Hilbert spaces or they are for accretive-type mappings in Banach spaces. Unfortunately, as has been rightly observed, many and probably most mathematical objects and models do not naturally live in Hilbert spaces. We have considered the class of generalized $\Phi$-strongly monotone mappings in Banach spaces, the class of monotone-type mappings such that if a solution of the equation 
$0 \in A x$ exists, it is necessarily unique. Therefore, our results have generalized the recent and important results of Aibinu and Mewomo [3], Chidume

and Idu [9] and Diop et al. [12]. Moreover, our techniques of proofs are of independent interest.

\section{Acknowledgement:}

The first author acknowledge with thanks the bursary and financial support from Department of Science and Technology and National Research Foundation, Republic of South Africa Center of Excellence in Mathematical and Statistical Sciences (DST-NRF CoE-MaSS) Doctoral Bursary. Opinions expressed and conclusions arrived at are those of the authors and are not necessarily to be attributed to the CoE-MaSS.

\section{References}

[1] H. A. Abass, F.U. Ogbuisi, O.T. Mewomo,Common solution of split equilibrium problem and fixed point problem with no prior knowledge of operator norm, U.P.B. Sci. Bull., Series A, 80(1) (2018), 175-190.

[2] M. O. Aibinu, O. T. Mewomo, Algorithm for Zeros of monotone maps in Banach spaces, Proceedings of Southern Africa Mathematical Sciences Association (SAMSA2016) Annual Conference, 21-24 November 2016, University of Pretoria, South Africa, pp. 35-44, (2017).

[3] M. O. Aibinu, O. T. Mewomo, Strong convergence theorems for strongly monotone mappings in Banach spaces, (Submitted to Hacettepe University J. Math \& Stat).

[4] Ya. Alber, Metric and generalized projection operators in Banach spaces: properties and applications, In: Kartsatos AG (ed) Theory and applications of nonlinear operators of accretive and monotone type, Marcel Dekker, New York, pp. 15-50, (1996).

[5] Y. Alber and I. Ryazantseva, Nonlinear Ill Posed Problems of Monotone Type, Springer, London, (2006).

[6] D. Aussel, J. N. Corvellec and M. Lassonde, Mean value property and subdifferential criteria for lower semicontinuous functions, Trans. Am. Math. Soc., 347, pp. 4147-4161, (1995). 
[7] D. Aussel, J. N. Corvellec and M. Lassonde, Subdifferential characterization of quasiconvexity and convexity, J. Convex Anal., 1, pp. 195-201, (1994).

[8] D. Aussel and M. Fabian, Single-directional properties of quasimonotone operators, Set-Valued Var. Anal., 21, pp. 617-626, (2013). DOI 10.1007/s11228-013-0253-4.

[9] C. E. Chidume and K. O. Idu, Approximation of zeros of bounded maximal monotone mappings, solutions of Hammerstein integral equations and convex minimization problems, Fixed Point Theory Appl., (97), (2016), DOI 10.1186/s13663-016-0582-8.

[10] I. Cioranescu,Geometry of Banach spaces, duality mappings and nonlinear problems, Kluwer Academic Publishers Group, Dordrecht, (1990).

[11] F. H. Clarke, Optimization and Nonsmooth Analysis, WileyInterscience, New-York, (1983).

[12] C. Diop, T. M. M. Sow, N. Djitte and C. E. Chidume, Constructive techniques for zeros of monotone mappings in certain Banach spaces, SpringerPlus, 383 (4), (2015), DOI 10.1186/s40064-015-1169-2.

[13] B. de Finetti, Sulle stratificazioni convesse, Annali di Matematica Pura ed Applicata, 30, pp. 173-183, (1949).

[14] A.P. Farajzadeh1, A. Karamian1 and S. Plubtieng, On the translations of quasimonotone maps and monotonicity, J. of Ineq. Appl., 1 (192), (2012).

[15] A. P. Farajzadeh, S. Plubtieng When a vector quasimonotone mapping is a vector monotone mapping, Optim Lett., 8, (2014), 2127-2134, (2014) DOI 10.1007/s11590-013-0716-4.

[16] W. Fenchel, Convex cones, sets and functions, Lecture Notes, Princeton University, Princeton, (1953).

[17] N. Hadjisavvas, Translations of quasimonotone maps and monotonicity, Appl. Math. Lett., 19, pp. 913-915, (2006).

[18] A. Hassouni, Quasi-monotone multifunctions; Applications to optimality conditions in quasi-convex programming, Numer. Funct. Anal. Optim., 13 (3-4), pp. 267-275, (1992). 
[19] A. Hassouni and A. Jaddar, Quasi-convex functions and applications to optimality conditions in nonlinear programming, Appl. Math. Lett., 14, pp. 241-244, (2001).

[20] Y. He, A relationship between pseudomonotone and monotone mappings, Appl. Math. Lett., 17, pp. 459-461, (2004).

[21] G. Isac and D. Motreanu, Pseudomonotonicity and quasimonotonicity by translations versus monotonicity in Hilbert spaces, Aust. J. Math. Anal. Appl., 1 (1), pp. 1-8, (2004).

[22] L. O. Jolaoso, F. U. Ogbuisi, O. T. Mewomo, An iterative method for solving minimization, variational inequality and fixed point problems in reflexive Banach spaces, Adv. Pure Appl. Math., 9 (3), pp. 167-184, (2017).

[23] L.O. Jolaoso, K.O. Oyewole, C. C. Okeke, O.T. Mewomo, A unified algorithm for solving split generalized mixed equilibrium problem and fixed point of nonspreading mapping in Hilbert space, Demonstr. Math., 51, pp. 211-232, (2018).

[24] S. Kamimura and W. Takahashi, Strong convergence of proximaltype algorithm in Banach space, SIAMJ Optim., 13 (3), pp. 938-945, (2002).

[25] S. Karamardian and S. Schaible, Seven kinds of monotone maps, J. Optim. Theory Appl., 66, pp. 37-46, (1990).

[26] S. Karamardian, S. Schaible and J.P Crouuzeix, Characterizations of generalized monotone maps, J. Optim. Theory Appl., 76, pp. 399-413, (1993).

[27] S. Karlin, Mathematical Methods and Theory in Games, Programming, and Economics, Pergamon Press, London, (1959).

[28] K. Kido, Strong convergence of resolvents of monotone operators in Banach spaces, Proc. Amer. Math. Soc., 103 (3), pp. 755-7588, (1988).

[29] V. L. Levin, Quasiconvex functions and quasimonotone operators, J. Conv. Anal., 2 (1-2), pp. 167-172, (1995).

[30] O.T. Mewomo and F.U. Ogbuisi, Convergence analysis of iterative method for multiple set split feasibility problems in certain Banach spaces, Quaest. Math., 41 (1), pp. 129-148, (2018). 
[31] F. U. Ogbuisi, O.T. Mewomo, Convergence analysis of common solution of certain nonlinear problems, Fixed Point Theory, 19 (1), pp. 335-358, (2018).

[32] J. P. Penot, Are generalized derivatives useful for generalized convex functions?, University of Pau, pp. 1-44, (1997).

[33] J. P. Penot and P. H. Quang, Generalized convexity of functions and generalized monotonicityof set-valued maps, J. Optim. Theory Appl., 92 (2), pp. 343-356, (1997).

[34] R. R. Phelps, Convex functions, monotone operators, and differentiability, 2nd edn. In: Lecture Notes in Math, vol. 1364. Springer-Verlag, Berlin, (1993).

[35] S. Reich, A weak convergence theorem for the alternating method with Bregman distances, In: A. G, Kartsatos (Ed.), Theory and Applications of nonlinear operators of accretive and monotone type, Lecture Notes Pure Appl. Math., Dekker, New York, 178, pp. 313-318, (1996). Anal. Appl. 75, pp. 287-292, (1980).

[36] R. T. Rockafellar, On the maximality of sums of nonlinear monotone operators, Trans. Amer. Math. Soc., 149, pp. 75-88, (1970).

[37] R. T. Rockafellar, Generalized directional derivatives and subgradients of nonconvex functions, Canad. J. Math., 32, pp. 257-280, (1980).

[38] H. K. Xu, Inequalities in Banach spaces with applications, Nonlinear Anal., 16 (12), pp. 1127-1138, (1991).

[39] H. K. Xu, Iterative algorithms for nonlinear operators, J. London Math. Soc., 66 (1), pp. 240-256, (2002).

[40] Z. B. Xu and G. F. Roach, Characteristic inequalities of uniformly convex and uniformly smooth Banach spaces, J. Math. Anal. Appl., 157, pp. 189-210, (1991).

[41] Y. Shehu and O.T. Mewomo, Further investigation into split common fixed point problem for demicontractive operators, Acta Math, Sin. (Engl. Ser.), 32 (11), pp. 1357-1376, (2016).

[42] C. Zălinescu, On uniformly convex functions, J. Math. Anal. Appl., 95, pp. 344-374, (1983). 


\section{O. Aibinu}

School of Mathematics,

Statistics and Computer Science,

University of KwaZulu-Natal, Durban, South Africa

e-mail : moaibinu@yahoo.com

and

\section{O. T. Mewomo}

School of Mathematics,

Statistics and Computer Science,

University of KwaZulu-Natal, Durban, South Africa

e-mail : mewomoo@ukzn.ac.za 